\title{
New Concepts in the Pathophysiology of Infective Endocarditis
}

\author{
Eleonora Widmer, $M D, P h D$, Yok-Ai Que, MD, PhD, \\ José M. Entenza, PhD, and Philippe Moreillon, MD, PhD
}

\begin{abstract}
Corresponding author Philippe Moreillon, MD, PhD

Department of Fundamental Microbiology, University of Lausanne, Biophore building, $\mathrm{CH}-\mathrm{IOI} 5$ Lausanne, Switzerland.

E-mail: philippe.moreillon@unil.ch
\end{abstract}

Current Infectious Disease Reports 2006, 8:27I-279

Current Science Inc. ISSN 1523-3847

Copyright () 2006 by Current Science Inc.

Endocarditis pathogens colonize valves with pre-existing sterile vegetations or valves with minimal endothelial lesions. Inflamed endothelia produce cytokines, integrins, and tissue factor, which in turn attract fibronectin, monocytes, and platelets. Bacteria attaching to such structures further activate the cascade, becoming embedded and protected from host defenses. Staphylococcus aureus also actively invade the endothelium, causing apoptosis and endothelial damage. Knowledge of this interplay identifies host factors as potential therapeutic targets. Blocking infection by modulating host factors might be opportune because host factors are conserved. In contrast, interfering with bacterial virulence factors might be more complicated because they vary among different bacteria.

\section{Introduction}

Despite improvements in health care, the incidence of infective endocarditis (IE) (2-6 per 100,000 population per year) has not changed over the past two decades [1,2]. This apparent paradox results from progressive modifications in risk factors. Chronic rheumatic heart disease is now rare in industrialized countries. This group of at-risk patients has been replaced with new at-risk patients, including intravenous drug users, elderly people with valve sclerosis, patients with intravascular prostheses, patients exposed to nosocomial bacteremia, and hemodialysis patients [1-3]. As a result, the mean age of patients with IE has increased from 30 years in the 1950s to older than 60 years since the 1990s. IE involves both host and microbial factors. The principal actors of this liaison dangereuse are reviewed.

\section{The Critical Role of Host Factors The valve endothelium}

The normal valve endothelium is very resistant to infection. However, mechanical lesions of this endothelium result in exposure of the underlying extracellular matrix proteins, local inflammation, and deposition of fibrin and platelets, which form a nonbacterial thrombotic endocarditis (NBTE) that is prone to bacterial colonization. Congenital cardiac abnormalities cause turbulent blood flow, which may provoke endothelial trauma. Valve scarring and calcification related to rheumatic carditis or valve sclerosis in elderly patients result in endothelial lesions. Degenerative valve lesions are detected in up to $50 \%$ of patients older than 60 years of age and may explain the increased risk for IE in the elderly [4]. Extrinsic intervention, such as prosthetic valve replacement or indwelling electrodes or catheters, also promotes endothelial lesions. In addition, Chlamydia pneumoniae or cytomegalovirus has been linked to atherosclerosis. Whether these conditions trigger endothelial lesions that promote IE is not known.

IE may also occur without identifiable pre-existing valve lesions. This is particularly true for Staphylococcus aureus, which has emerged as the leading cause of IE in recent surveys $[1,2]$. Local inflammation, which may occur in some circumstances, triggers endothelial cells to express a variety of molecules, including integrins of the $\beta 1$ family (very late antigen). Integrins are transmembrane proteins that can connect extracellular determinants to the cellular cytoskeleton. Integrins of the $\beta 1$ family bind circulating fibronectin to the endothelial surface. $S$. aureus and a few other IE pathogens carry fibronectin-binding proteins anchored to their walls. Thus, fibronectin bound by activated endothelia provides an adhesive surface to these circulating bacteria.

\section{Inflammation and coagulation}

Inflammation and coagulation are very closely related. Tissue factor (TF), which is a main player in coagulation and vegetation growth, is induced from monocytes and endothelial cells by interleukin (IL)-1 [5], which is itself produced in response to tissue injury. In turn, endothelial cells and monocytes produce proinflammatory cytokines and integrins that favor the construction of the NBTE 
and thus the attachment of circulating bacteria. Once attached, microbes further maintain this vicious cycle that promotes growth of the vegetation, in which they are protected from host defenses. A synthetic view of this complex cycle is starting to emerge. However, although microbial characteristics may change according to the infecting species, the host counterparts are conserved. Therefore, critical host components could be convenient targets for adjuvant interventions to antibiotherapy.

\section{Tissue factor}

TF is a procoagulant integral membrane glycoprotein of $47 \mathrm{kD}$. It is produced on the surface of virtually all cells except for unperturbed endothelia and circulating blood cells [5]. When endothelial damage occurs, TF from underlying tissues reacts with factor VII and factor $\mathrm{X}$, which cleave prothrombin to thrombin, which triggers the polymerization of fibrinogen into fibrin. TF also activates platelets, which are integral components of the vegetation [6]. Although unperturbed endothelia and monocytes do not produce $\mathrm{TF}$, they can be induced to produce it by various agonists, including cytokines (IL1) and bacterial products [5]. Intravegetation TF activity (TFA) was demonstrated in specimens from experimental IE due to Streptococcus sanguis, S. aureus, and Staphylococcus epidermidis, and the presence of TFA was associated with the presence of the microbes $[7,8]$.

Induction of TFA from the host monocytes

Monocytes are present in the early vegetation, and their binding to fibrin clots elicits TFA in vitro [9]. Moreover, addition of $S$. sanguis to the system increases TFA approximately 10 -fold. This occurred in spite of the fact that monocytes could not physically engulf and remove fibrin-adherent streptococci-a phenomenon referred to as "frustrated phagocytosis"-suggesting that some extrinsic bacterial factor was involved. Such microbial factors interacting with innate host defenses have been referred to as modulins. S. aureus and S. epidermidis can also induce TFA from monocytes $[7,8]$. Moreover, the contribution of monocytes to intravegetation TFA was confirmed in animals with etoposide-induced monocytopenia $[7,8]$. Thus, bacteria can encourage vegetation growth by subverting monocytes to produce TFA.

\section{Induction of TFA from the host endothelium}

Because $S$. aureus IE might develop on physically intact endothelia, the question arose as to whether these organisms can trigger local vegetation formation by direct induction of endothelial TFA. Veltrop et al. [7] showed that $S$. aureus, but not $S$. epidermidis and S. sanguis, could induce TFA expression from cultured endothelial cells. Treating these cells with IL-1 also induced TFA. However, abrogating the IL-1 response with IL-1-receptor antagonist did not abrogate TFA induction by $S$. aureus. Thus, IL-1 and $S$. aureus induce endothelial TFA by distinct mechanisms.
Induction of endothelial TFA with S. epidermidis and $S$. sanguis needed the additional presence of monocytes [10]. This required endothelial expression of intercellular adhesion molecule 1 (ICAM-1) and vascular cell adhesion molecule 1 (VCAM-1), which depend on preexisting endothelial activation. Therefore, although $S$. aureus and IL-1 might be enough to initiate endothelial procoagulant activity, S. epidermidis and S. sanguis depend on a pre-existing endothelial stress (or damage) to trigger the same effect.

\section{Cytokines}

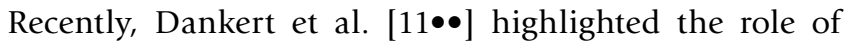
IL-1 $\alpha$ in maintaining endothelial inflammation. In experimental endocarditis, the initial valve lesion is produced via a catheter inserted through the valve leaflets and left in place throughout the experiment. Bacteria injected thereafter infect the damaged valves. The authors observed that removal of the catheter 24 hours before bacterial challenge was enough to restore resistance to infection, presumably due to spontaneous endothelial repair. However, animals from which the catheter had been removed could be rendered susceptible to infection again by injection of IL- $1 \alpha 3$ hours prior to bacterial challenge. This 3 -hour window corresponded to the time required for IL-1 to induce maximal TFA from endothelial cells in vitro. Thus, IL-1 $\alpha$ could resensitize endothelia with minimal lesions to infection susceptibility. Systemic inflammation could be important in moderate valve anomalies as in early degenerative lesions, or following illicit drug injection where impurities are thought to produce minimal lesions to the leaflets' endothelium. Concurrent inflammation due to unrelated diseases could increase susceptibility to IE in these conditions. It would be important to know whether IL-1 $\alpha$ has the same effect on nonprimed endothelia, but this is as yet uncertain.

\section{The platelets paradox}

Bacterial-induced platelet aggregation is commonly considered to be a pathogenic factor. However, platelets also contribute to anti-infective host defenses by releasing antimicrobial peptides and inflammatory mediators [6]. Platelets harbor three types of granules $(\alpha, \delta$, and $\lambda)$, which release a variety of mediators involved in adhesion and coagulation ( $\alpha$ granules), vascular tone ( $\delta$ granules), and thrombus dissociation ( $\lambda$ granules) [6]. $\alpha$ Granules also contain an arsenal of antimicrobial peptides, collectively called platelet microbicidal proteins (PMPs), in rabbit platelets and thrombocidins in human platelets. PMPs represent one of the first host-defense barriers in IE, and are released upon platelet stimulation, for instance by thrombin.

\section{Pathogenic role in streptococcal IE}

$S$. sanguis has the ability to induce platelet aggregation via two surface-expressed antigens [12]. The class I antigen promotes streptococcal adhesion to platelets $\left(\mathrm{Adh}^{+}\right.$ 
Table I. Potential Staphylococcus aureus MSCRAMMs belonging to the LPXTG-anchoring domain family of wall-associated proteins

\begin{tabular}{|c|c|c|c|}
\hline Gene & Protein & Function & Potential implication in disease \\
\hline \multirow[t]{2}{*}{ spa } & Protein A & Binds antibody Fc fragment & Experimental sepsis \\
\hline & & & Experimental osteoarthritis \\
\hline clfA & Clumping factor $\mathrm{A}$ & Binding to fibrinogen and platelets & Experimental endocarditis \\
\hline clfB & Clumping factor B & Binding to fibrinogen & Not proven in endocarditis \\
\hline cna & Collagen-binding protein & Binding to collagen & Experimental arthritis \\
\hline \multirow[t]{2}{*}{ fnbA } & Fibronectin-binding protein A & $\begin{array}{l}\text { Binding to fibronectin, } \\
\text { fibrinogen, elastin }\end{array}$ & Experimental endocarditis \\
\hline & & Activates platelets & Cell invasion \\
\hline \multirow[t]{2}{*}{$f n b B$} & Fibronectin-binding protein B & Binding to fibronectin and fibrinogen & Undetermined \\
\hline & & Activates platelets & \\
\hline$s d r C$ & Serine-aspartate repeat protein & Binding to fibrinogen & Undetermined \\
\hline$s d r D$ & Serine-aspartate repeat protein & Possible binding to fibrinogen & Undetermined \\
\hline sdrE & Serine-aspartate repeat protein & Possible binding to fibrinogen & Undetermined \\
\hline pls & Plasmin-sensitive protein & Binding to nasal mucosal cells & Colonization of nasal mucosa \\
\hline$f m t B$ & $\begin{array}{l}\text { Factor affecting methicillin resistance } \\
\text { in the presence of Triton X-100 }\end{array}$ & Putative cell-wall building & $\begin{array}{l}\text { Affects the expression of } \\
\text { methicillin resistance }\end{array}$ \\
\hline sasA (sraP) & S. aureus surface protein $A$ & Platelet binding & Possible in experimental endocarditis \\
\hline$s a s B$ & S. aureus surface protein B & Undetermined & Undetermined \\
\hline sasC & S. aureus surface protein C & Undetermined & Undetermined \\
\hline sasE & S. aureus surface protein $E$ & Implicated in transferring binding & Undetermined \\
\hline sasF & S. aureus surface protein $\mathrm{F}$ & Undetermined & Undetermined \\
\hline sasG & S. aureus surface protein G & Binding to nasal mucosal cells & Associated with invasive disease \\
\hline sasH & S. aureus surface protein $\mathrm{H}$ & Undetermined & Associated with invasive disease \\
\hline sasl & S. aureus surface protein I & Undetermined & Undetermined \\
\hline sas] & S. aureus surface protein J & Implicated in transferring binding & Undetermined \\
\hline sask & S. aureus surface protein $\mathrm{K}$ & Undetermined & Undetermined \\
\hline
\end{tabular}

MSCRAMMs - microbial surface component reacting with adhesive matrix molecules.

(Adapted from Siboo et al. [18], Que et al. [40••], and Roche et al. [44].)

phenotype). The class II antigen triggers further platelet aggregation ( $\mathrm{Agg}^{+}$phenotype). This so-called platelet aggregation-associated protein contains a collagen-like platelet-interacting domain [12]. Studies indicated that an $\mathrm{Agg}^{+}$isolate of $S$. sanguis produced more severe infection and larger vegetations than an $\mathrm{Agg}^{-}$control strain in experimental IE [13]. However, other studies did not find such a correlation. In these studies the $\mathrm{Agg}^{-}$comparator strains were not characterized for other pro-endocarditis factors, or were less adherent to fibronectin, fibrinogen, and platelet-fibrin clots [13]. Moreover, not all IE streptococcal isolates are $\mathrm{Agg}^{+}$, indicating that additional factors are involved [14].

Streptococcal-platelet binding was further characterized in Streptococcus mitis [15]. Binding was mediated by a complex interaction between at least three loci, $p b l A, p b l B$, and pblT. PblA and PblB are surface structures that are likely to act as adhesins. They are encoded by a lysogenic bacteriophage (SM1) of S. mitis, suggesting that they might disseminate to other pathogens. PblT is homologous to a membrane transporter of the major facilitator superfamily. Solute-binding proteins of such transport systems have been shown to mediate adherence. However, mutants in these loci have not been tested in experimental IE.

\section{Pathogenic role in staphylococcal IE}

S. aureus organisms bind platelets directly or indirectly via several surface proteins, including fibrinogen-binding and fibronectin-binding proteins, as well as a newly described serine-rich protein called SraP (Table 1) [1619]. Adhesion of $S$. aureus to activated platelets may also occur via thrombospondin, von Willebrand factor, protein A, and/or C1q bridging. 
Sullam et al. [20] compared an $\mathrm{Agg}^{+}$parent S. aureus to a Tn551-insertional mutant with decreased plateletbinding and aggregation in experimental endocarditis. The parent strain was up to 100 -fold more infective than the mutant, as determined 48 hours after challenge. The organisms were comparable for other relevant virulence properties, including their susceptibility to platelet peptide-induced killing, and their ability to bind to fibrinogen and fibronectin. This provides strong evidence for the implication of $S$. aureus-induced platelet aggregation in IE pathogenesis. The same laboratory showed that fibrinogen-binding protein A (ClfA) was involved in this interaction.

\section{The antimicrobial effects of platelets}

Conversely, experimental IE due to an $S$. sanguis strain was more severe in thrombocytopenic rabbits than in control animals with normal platelet counts [21]. The infecting organism extensively aggregated platelets, but was rapidly killed by PMPs in vitro. Dankert et al. [22] confirmed the role of PMPs in experimental IE by using a series of PMP-susceptible and PMP-resistant streptococcal isolates. PMP-susceptible streptococci colonized more effectively damaged valves than PMP-resistant strains 5 minutes after bacterial challenge. However, they were rapidly eradicated from the vegetations during the following 24 to 48 hours, whereas PMP-resistant strains persisted. Therefore, although bacteria may subvert platelets to develop the vegetation, they need to resist PMP-induced killing to take advantage of this procoagulant effect.

The mechanism of action of PMP was clarified in $S$. aureus. Thrombin-induced PMP-1 (tPMP-1), the principal PMP released from rabbit platelets, is a cationic molecule of relatively small size $(8.036 \mathrm{Da})$. It permeabilizes the cytoplasmic membrane in a voltage-dependent manner without classic pore formation [23], which is different from other types of bactericidal peptides. Recently, resistance to PMP-induced killing was associated with the dlt $A B C$ and $m r p F$ loci, the products that are responsible for alanylation of surface teichoic and lipoteichoic acids, and lysinylation of membrane phosphatidyl glycerol [24]. Both of these surface structures are positively charged. They are likely to repel the cationic PMPs and, thus, protect the bacterium.

\section{Clinical implication of PMPs}

Experimental studies support the protective role of PMPs in intravascular infections $[21,22]$. In humans, $S$. aureus isolates from intravascular infections were more often resistant to PMPs than those isolated from other types of infections [25]. PMPs may also play a role in therapy. tPMP-1 acted synergistically with several antibiotics-in particular, cell-wall active drugs-both in vitro and in vivo [14], and the effect was observed against both PMPsusceptible and PMP-resistant isolates. Thus, development of PMP analogues for therapy may be envisioned.
Because platelets are involved in IE, investigators explored the effect of adjuvant antiplatelet therapy in the disease. Limited clinical observations suggest that aspirin might prevent vegetation growth and decrease cerebral septic emboli in human IE [26]. Nicolau et al. [27] studied the effect of aspirin and ticlopidine, two antiplatelet drugs with different modes of action, in rabbits with $S$. aureus experimental IE. Both drugs synergistically decreased the vegetation size, and also moderately decreased vegetation bacterial titers. However, the PMP-resistance phenotype of the infecting organism was not reported.

Aspirin also significantly decreased vegetation weight, vegetation bacterial titers, and renal septic emboli in $S$. aureus experimental IE [28]. In these experiments, preexposure of platelets to aspirin and preexposure of $S$. aureus to salicylic acid (the principal metabolite of aspirin in vivo) decreased platelet adherence and aggregation as well as bacterial adherence to fibrin and fibrin-platelet matrices in vitro. One reason is that salicylic acid can block the expression of global regulator agr, thus altering the expression of S. aureus pathogenic factors [28]. Thus, aspirin acts both on the platelets and on the microbe. Experiments also indicated that aspirin, but not ticlopidine, was synergistic with vancomycin in increasing valve sterilization during experimental therapy. Whether this is also true for streptococci is as yet not determined. However, although platelet antiaggregants have a potential efficacy, extrapolating these experimental results to the human situation must be tempered by the risk of hemorrhage related to anticoagulation during acute IE [29].

\section{The role of transient bacteremia}

Medicosurgical procedures in nonsterile anatomic sites may provoke transient bacteremia. However, mastication and tooth brushing do so as well $[30,31]$. The duration of bacteremia after dental manipulation is short $(\leq 15$ minutes), and its magnitude is not very different during various dental procedures compared to normal activity [31]. It was reported to be 1 to 10 colony-forming units (CFU) $/ \mathrm{mL}$ versus $10 \mathrm{CFU} / \mathrm{mL}$ during mastication and dental extraction, respectively [30]. It was estimated that the cumulative numbers of circulating bacteria (in CFU/ $\mathrm{mL} / \mathrm{y}$ ) resulting from 1) mastication, 2) tooth brushing two times per day, and 3) dental examination were 5.6 million times, 154,000 times, and 48 times greater, respectively, than that provoked by a single tooth extraction [32]. This probably explains why most cases of IE are not preceded by medicosurgical procedures. Moreover, if the cumulative exposure to circulating bacteria is a greater risk factor for IE than the magnitude of bacteremia during dental procedures, then the recommendation for antibiotic prophylaxis should be revisited. This issue remains to be solved.

Besides bacteremia of dental origin, two increasingly frequent "health care-associated" bacteremia are of concern: bacteremia in hemodialysis patients and nosocomial 
bacteremia. IE is up to three times more frequent in hemodialysis patients than in the general population [3] and more than $50 \%$ of the cases are due to S. aureus. Nosocomial IE accounted for $22 \%$ of 109 cases in one study [2]. Many patients had debilitating conditions, but less than $50 \%$ had known cardiac predisposing factors. The predominant pathogens were staphylococci and enterococci, which were frequently associated with catheters and/or medicosurgical procedures, and mortality was more than $50 \%$ [2]. It was estimated that more than $10 \%$ of nosocomial S. aureus bacteremia was responsible for subsequent IE. Therefore, these new conditions are important.

\section{Microbial Factors}

S. aureus, Streptococcus spp., and enterococci are responsible for more than $80 \%$ of all IE cases $[1,2]$. These organisms possess surface adhesins that mediate attachment to the vegetation and may modulate inflammation. These adhesins are collectively referred to as MSCRAMMs (microbial surface component reacting with adhesive matrix molecules) [33].

\section{MSCRAMMs of viridans streptococci}

Some streptococcal MSCRAMMs that were studied in experimental IE were reviewed [14]. Glucans are surface polysaccharides produced from dietary sucrose by bacterial glucosyltransferase (Gtf) and fructosyltransferase (Ftf) enzymes. Glucan production increases streptococcal adherence to damaged valves and fibrin-platelet clots, and triggers the production of IL-6 in vitro [34]. MSCRAMMs' role in experimental IE was demonstrated by using conditions favoring or not favoring the production of these surface polysaccharides, as well as in $g t f$ and $f t f$ inactivated mutants of Streptococcus mutans. However, $g t f$ inactivation did not decrease experimental infectivity in all streptococci, indicating that glucans are not uniformly required.

FimA is a pathogenic factor in Streptococcus parasanguis. Its inactivation greatly decreased the ability of $S$. parasanguis to adhere to immobilized fibrin, and to cause IE in rats. Moreover, immunization of animals with FimA conferred protection against subsequent experimental IE with the original strain [35]. FimA belongs to a family of oral mucosal adhesins, including SsaB of S. sanguis, ScaA of Streptococcus gordonii, PsaA of Streptococcus pneumoniae, and EfaA of Enterococcus faecalis. FimA-like proteins were found by cross-hybridization in a number of streptococci, suggesting that immunization could confer cross-protection against several organisms. Further experiments are required to confirm this hypothesis.

\section{MSCRAMMs of $S$. aureus}

S. aureus is extremely well equipped with both MSCRAMMs and secreted factors that mediate tissue colonization and invasion. Most described MSCRAMMs (Table 1) are cova- lently attached to the peptidoglycan via sortase processing of LPXTG proteins [36]. Other protein MSCRAMMs are loosely attached to the wall, including coagulase, extracellular adherence protein [37], and the recently described autolysin Aaa [38]. Nonprotein adhesins that promote experimental IE include teichoic acids, which mediate binding to platelets and endothelial cells $[24,39]$.

This intricate context makes it difficult to analyze the pathogenic role of individual gene products by classic gene-inactivation and gene-complementation experiments. To circumvent this problem, systems were developed to express individual staphylococcal determinants in surrogate bacteria missing the rest of the redundant staphylococcal environment. Using a system developed in Lactococcus lactis, Que et al. [40••] demonstrated that $S$. aureus ClfA was necessary and sufficient for early valve colonization and infection in rats with experimental IE, but not sufficient for invasive and persistent disease. Lactococci equipped with ClfA had a more than 100 -fold increased ability to colonize damaged valve over the control parent. However, they were progressively eradicated from the vegetations within 72 hours [40••]. This spontaneous healing is not characteristic of IE and was most likely due to the susceptibility of L. lactis to PMP-induced killing.

In the same study, fibronectin-binding protein $\mathrm{A}$ (FnBPA) promoted both early valve colonization and persistent infection. FnBPA is a peculiar MSCRAMM carrying at least three binding specificities-to fibronectin, to fibrinogen, and to elastin [41-43]. Constructing truncated proteins expressing only specific FnBPA-binding regions confirmed that fibrinogen binding was necessary and sufficient for early valve colonization, as observed with ClfA. In addition, fibronectin binding was necessary for further invasion and persistence. This invasive phenotype was associated with the capacity of fibronectin binding to trigger active internalization of staphylococci into eukaryotic cells, both in vitro and in vivo [40••]. However, truncated FnBPA expressing only fibronectin binding was unable to colonize damaged valves at first, and required the simultaneous expression of fibrinogen binding to provoke infection [40••]. Moreover, cooperation between fibrinogen-binding and fibronectin binding could occur both in cis, when the two domains were present on the same protein as in FnBPA, and in trans, when both fibrinogen-binding and fibronectin-binding domains were present on two separate surface molecules simultaneously expressed by separate plasmids in lactococci $[40 \bullet \bullet]$. Thus, interadhesin cooperation might also occur between other MSCRAMMs, adding even more flexibility to the already wealthy set of $S$. aureus surface determinants.

\section{Role of other protein-MSCRAMMs in S. aureus IE} S. aureus staphylococci carry up to 21 LPXTG-attached surface proteins, of which 10 have a known adhesin function 


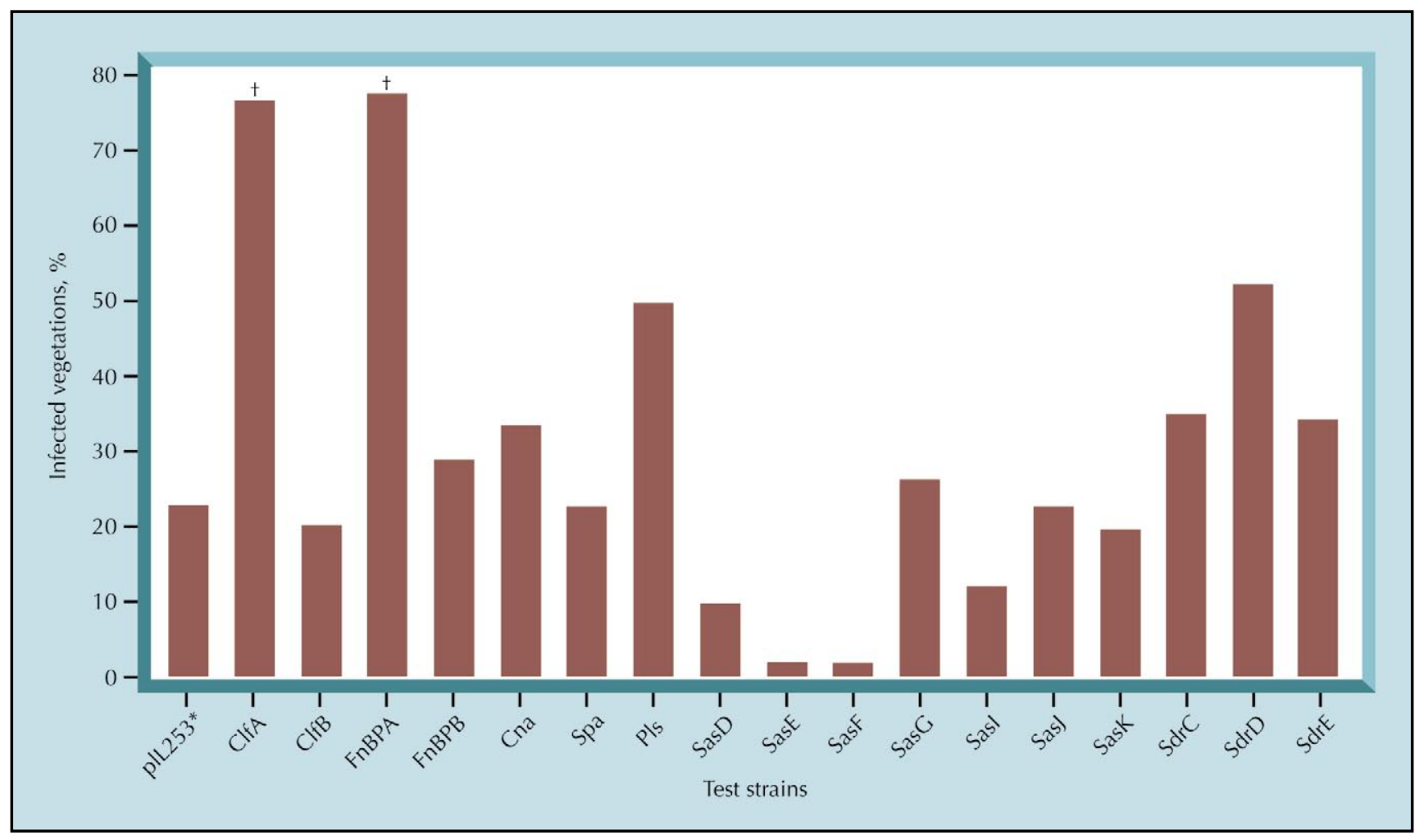

Figure 1. Induction of experimental endocarditis with lactococcal recombinants expressing 17 of the 21 LPXTG wall-attached proteins of Staphylococcus aureus (Table 1). Groups of 10 rats with catheter-induced aortic vegetations were challenged with $10^{6}$ colony-forming units of each of the recombinant organisms, and the proportion of valve infection was assessed 16 hours later. The test strains are indicated at the bottom of each column. plL253 (asterisk) stands for the control Lactococcus lactis carrying an empty expression vector. The other 17 abbreviations represent the name of the staphylococcal protein expressed by each of the tested recombinants. Daggers indicate that the percentage of infected vegetations was statistically different from that of the pIL253 control strain $(P<0.05)$ [47].

(Table 1) [44]. In ongoing work (Widmer et al., Unpublished data), we succeeded in cloning and expressing 17 of them in the lactococcal system (Fig. 1). Protein expression was assessed by mass spectrometry and the recombinant lactococci were tested for in vitro adherence and in vivo infection in rats with experimental IE (Fig. 1). Adherence tests confirmed known properties of ClfA, ClfB, FnBPA, FnBPB, and collagen-binding protein (Cna). In addition, new properties were detected, such as adherence to fibrinogen by plasmin-sensitive protein (Pls), and adherence to keratin by ClfA, FnBPA, and Sdr. However, in spite of these functional redundancies, only ClfA and FnBPA significantly $(P<0.05)$ promoted infection in experimental IE (Fig. 1).

It was surprising that $\mathrm{ClfB}$ and FnBPB, which both bind fibrinogen, did not promote valve infection. One hypothesis is that their fibrinogen-binding sites became saturated by soluble fibrinogen in the plasma before binding to the vegetation [45]. It is also relevant that ClfB did not affect experimental IE in S. aureus-deleted mutants. Nevertheless, although the contribution of other adhesins is not entirely excluded, these results underline the critical role of ClfA and FnBPA in endovascular infection.

\section{Role of gene regulation}

The expression of surface and secreted factors is orchestrated by the global regulators agr (accessory gene regulator), sar (staphylococcal accessory regulator), and other determinants, including sigB [46]. Together, sar and agr coordinately regulate the expression of adhesins during exponential growth and the secretion of soluble factors in the postexponential growth phase in vitro. Moreover, they were shown to be critical for infectivity in experimental IE and other models in vivo [47,48].

\section{Regulation of adhesions}

Inactivating agr alone decreased pathogenicity in experimental-model subcutaneous abscesses, where exoprotein production is likely to be important [48]. Nevertheless, agr inactivation did not have much influence on the course of experimental endocarditis, where bacterial surface adhesins are critical for valve colonization [47]. The logic to this result is that agr inhibits adhesin production in the late exponential phase, whereas the negative mutant does not. Therefore, although the negative mutant is hampered in exoprotein production, it is still fully equipped with surface-bound colonizing determinants. The effect of agr deletion could be partially restored by concomitant activation of the reciprocal regulator rot, thus highlighting the complexity of the network.

However, inactivating sar decreased infectivity in experimental IE because it also decreased the expression of surface adhesins [47], whereas overexpression of 
sigmaB increased early infectivity because it increased the expression of fibrinogen binding [49]. This further underlines the role of fibrinogen binding in IE induction.

\section{Regulation of exoproteins}

Regulation of adhesin expression is important because it affects colonization. However, regulation of exoproteins and toxin expression is critical as well. As mentioned, FnBPA-positive lactococci or $S$. aureus can invade perivegetation endothelial cells after colonization. After endothelium invasion, the recombinant lactococci can divide, but mostly remained trapped inside the cells. In contrast, after internalization, $S$. aureus can lyse the cells and spread further $[40 \bullet \bullet]$.

Cell lysis is mediated by hemolysins, which are positively regulated by agr [46]. Yet, although hemolysins are useful for escaping the host cell, they are dangerous when the bacteria become exposed to blood cells. Staphylococcal mutants overexpressing $\alpha$-hemolysin produced less severe experimental IE than did parent strains, presumably because they lysed and activated platelets, which consequently released PMPs [50]-thus, the importance of hemolysin regulation, as hemolysins are only expressed at high cell density and are turned off in scattered growing cells [46].

\section{Conclusions}

IE is an exquisite model of bacteria-host interactions. At first glance, it is seems simple. Microbes equipped with specific adhesins can stick to pre-existing endovascular thrombi, in which they get embedded and are protected from host defenses. However, digging into these mechanisms indicates that the microbes are equipped with an array of determinants that do not only attach to extracellular matrix molecules but also directly to endothelia and platelets, which become activated and promote vegetation formation and growth. These interactions involve serial reactions with host components, including fibrinogen, fibronectin, von Willebrand factor, complement, and antibodies in the case of platelet activation. Bacteria can also invade endothelial cells, where they can either reside, thus evading host defenses and antibiotics, or escape to spread further after having lysed the host cell. Surviving the intracellular and extracellular milieus requires tight regulation of an array of pathogenic genes that are aimed at specific environmental-dependent tasks.

On the side of the host, monocytes, endothelial cells, and platelets coordinately reunify both healing and antimicrobial processes. It is likely that these reactions successfully abort numerous episodes of bacteremia and early valve colonization. This could explain the inoculum dependency of IE, where a threshold number of bacteria is required for successful infection. However, these healing and defense systems can also become subverted by the bacteria.
Current interventions against IE primarily target the microbes. Although this is sound, knowing the host players that are subverted might help identify parallel targets of the host components. These have the advantage of being conserved, as opposed to microbial factors that vary depending on the infecting organisms. They will include modulating inflammation, expression of intercellular adhesion molecules, and platelet functions.

\section{Acknowledgments}

This work was supported by grants \#3200-65371.01 and 3200-65371/2 from the Swiss National Fund for Scientific Research, and a grant from the Helmut Horten Foundation to E. Widmer.

\section{References and Recommended Reading}

Papers of particular interest, published recently,

have been highlighted as:

- Of importance

-• Of major importance

1. Moreillon P, Que YA: Infective endocarditis. Lancet 2004, 363:139-149.

2. Bouza E, Menasalvas A, Munoz P, et al.: Infective endocarditis-a prospective study at the end of the twentieth century: new predisposing conditions, new etiologic agents, and still a high mortality. Medicine (Baltimore) 2001, 80:298-307.

3. Abbott KC, Agodoa LY: Hospitalizations for bacterial endocarditis after initiation of chronic dialysis in the United States. Nephron 2002, 91:203-209.

4. Croft LB, Donnino R, Shapiro R, et al.: Age-related prevalence of cardiac valvular abnormalities warranting infectious endocarditis prophylaxis. Am J Cardiol 2004, 94:386-389.

5. Camerer E, Kolsto AB, Prydz H: Cell biology of tissue factor, the principal initiator of blood coagulation. Thromb Res 1996, 81:1-41.

6. Yeaman MR: The role of platelets in antimicrobial host defense. Clin Infect Dis 1997, 25:951-968.

7. Veltrop MH, Beekhuizen H, Thompson J: Bacterial species- and strain-dependent induction of tissue factor in human vascular endothelial cells. Infect Immun 1999, 67:6130-6138.

8. Bancsi MJ, Veltrop MH, Bertina RM, et al.: Role of monocytes and bacteria in Staphylococcus epidermidis endocarditis. Infect Immun 1998, 66:448-450.

9. Bancsi MJ, Thompson J, Bertina RM: Stimulation of monocyte tissue factor expression in an in vitro model of bacterial endocarditis. Infect Immun 1994, 62:5669-5672.

10. Veltrop MH, Thompson J, Beekhuizen H: Monocytes augment bacterial species- and strain-dependent induction of tissue factor activity in bacterium-infected human vascular endothelial cells. Infect Immun 2001, 69:2797-2807.

11.• Dankert J, van der Werff J, Joldersma W, et al.: Interleukin 1alpha increases the susceptibility of rabbits to experimental viridans streptococcal endocarditis. Infect Immun 2006, 74:947-952.

Describes the potential of circulating interleukin- $1 \alpha$ for triggering inflammation of minimally altered valve endothelia, thus making them susceptible to bacterial colonization. 
12. Erickson PR, Herzberg MC: The Streptococcus sanguis platelet aggregation-associated protein. Identification and characterization of the minimal platelet-interactive domain. J Biol Chem 1993, 268:1646-1649.

13. Manning JE, Hume EB, Hunter N, et al.: An appraisal of the virulence factors associated with streptococcal endocarditis. J Med Microbiol 1994, 40:110-114.

14. Moreillon P, Que YA, Bayer AS: Pathogenesis of streptococcal and staphylococcal endocarditis. Infect Dis Clin North Am 2002, 16:297-318.

15. Bensing BA, Siboo IR, Sullam PM: Proteins PblA and PblB of Streptococcus mitis, which promote binding to human platelets, are encoded within a lysogenic bacteriophage. Infect Immun 2001, 69:6186-6192.

16. Fitzgerald JR, Loughman A, Keane F, et al.: Fibronectinbinding proteins of Staphylococcus aureus mediate activation of human platelets via fibrinogen and fibronectin bridges to integrin GPIIb/IIIa and IgG binding to the FcgammaRIIa receptor. Mol Microbiol 2006, 59:212-230.

17. Loughman A, Fitzgerald JR, Brennan MP, et al.: Roles for fibrinogen, immunoglobulin and complement in platelet activation promoted by Staphylococcus aureus clumping factor A. Mol Microbiol 2005, 57:804-818.

18. Siboo IR, Chambers HF, Sullam PM: Role of SraP, a serinerich surface protein of Staphylococcus aureus, in binding to human platelets. Infect Immun 2005, 73:2273-2280.

19. Bayer AS, Sullam PM, Ramos M, et al.: Staphylococcus aureus induces platelet aggregation via a fibrinogendependent mechanism which is independent of principal platelet glycoprotein IIb/IIIa fibrinogen-binding domains. Infect Immun 1995, 63:3634-3641.

20. Sullam PM, Bayer AS, Foss WM, et al.: Diminished platelet binding in vitro by Staphylococcus aureus is associated with reduced virulence in a rabbit model of infective endocarditis. Infect Immun 1996, 64:4915-4921.

21. Sullam PM, Frank U, Yeaman MR, et al.: Effect of thrombocytopenia on the early course of streptococcal endocarditis. J Infect Dis 1993, 168:910-914.

22. Dankert J, Van den Werff J, Zaat SAJ, et al.: Involvement of bactericidal factors from thrombin-stimulated platelets in clearance of adherent viridans streptococci in experimental infective endocarditis. Infect Immun 1995, 63:663-671.

23. Koo SP, Bayer AS, Kagan BL, et al.: Membrane permeabilization by thrombin-induced platelet microbicidal protein 1 is modulated by transmembrane voltage polarity and magnitude. Infect Immun 1999, 67:2475-2481.

24. Weidenmaier C, Peschel A, Kempf VA, et al.: DltABCD- and MprF-mediated cell envelope modifications of Staphylococcus aureus confer resistance to platelet microbicidal proteins and contribute to virulence in a rabbit endocarditis model. Infect Immun 2005, 73:8033-8038.

25. Fowler VG Jr, McIntyre LM, Yeaman MR, et al.: In vitro resistance to thrombin-induced platelet microbicidal protein in isolates of Staphylococcus aureus from endocarditis patients correlates with an intravascular device source. J Infect Dis 2000, 182:1251-1254.

26. Taha TH, Durrant SS, Mazeika PK, et al.: Aspirin to prevent growth of vegetations and cerebral emboli in infective endocarditis. J Intern Med 1992, 231:543-546.

27. Nicolau DP, Tessier PR, Nightingale CH: Beneficial effect of combination antiplatelet therapy on the development of experimental Staphylococcus aureus endocarditis. Int $J$ Antimicrob Agents 1999, 11:159-161.

28. Kupferwasser LI, Yeaman MR, Nast CC, et al.: Salicylic acid attenuates virulence in endovascular infections by targeting global regulatory pathways in Staphylococcus aureus. J Clin Invest 2003, 112:222-233.

29. Tornos P, Almirante B, Mirabet S, et al.: Infective endocarditis due to Staphylococcus aureus: deleterious effect of anticoagulant therapy. Arch Intern Med 1999, 159:473-475.

30. Mc Entergart MG, Porterfield JS: Bacteremia following dental extraction. Lancet 1949, 2:596-598.
31. Hall G, Heimdahl A, Nord CE: Bacteremia after oral surgery and antibiotic prophylaxis for endocarditis. Clin Infect Dis 1999, 29:1-8; quiz 9-10.

32. Bahn SL, Goveia G, Bitterman P, et al.: Experimental endocarditis induced by dental manipulation and oral streptococci. Oral Surg Oral Med Oral Pathol 1978, 45:549-559.

33. Patti JM, Allen BL, McGavin MJ, et al.: MSCRAMM-mediated adherence of microorganisms to host tissues. Annu Rev Microbiol 1994, 48:585-617.

34. Shun CT, Lu SY, Yeh CY, et al.: Glucosyltransferases of viridans streptococci are modulins of interleukin- 6 induction in infective endocarditis. Infect Immun 2005, 73:3261-3270.

35. Viscount HB, Munro CL, Burnette-Curley D, et al.: Immunization with FimA protects against Streptococcus parasanguis endocarditis in rats. Infect Immun 1997, 65:994-1002.

36. Navarre WW, Schneewind O: Surface proteins of grampositive bacteria and mechanisms of their targeting to the cell wall envelope. Microbiol Mol Biol Rev 1999, 63:174-229.

37. Palma M, Haggar A, Flock JI: Adherence of Staphylococcus aureus is enhanced by an endogenous secreted protein with broad binding activity. J Bacteriol 1999, 181:2840-2845.

38. Heilmann C, Hartleib J, Hussain MS, et al.: The multifunctional Staphylococcus aureus autolysin aaa mediates adherence to immobilized fibrinogen and fibronectin. Infect Immun 2005, 73:4793-4802.

39. Weidenmaier C, Peschel A, Xiong YQ, et al.: Lack of wall teichoic acids in Staphylococcus aureus leads to reduced interactions with endothelial cells and to attenuated virulence in a rabbit model of endocarditis. J Infect Dis 2005, 191:1771-1777.

40.• Que YA, Haefliger JA, Piroth L, et al.: Fibrinogen and fibronectin binding cooperate for valve infection and invasion in Staphylococcus aureus experimental endocarditis. J Exp Med 2005, 201:1627-1635.

Discloses the critical molecular cooperation between $S$. aureus surface proteins binding to fibrinogen and fibronectin for valve colonization and endothelial invasion.

41. Roche FM, Downer R, Keane F, et al.: The N-terminal A domain of fibronectin-binding proteins $\mathrm{A}$ and $\mathrm{B}$ promotes adhesion of Staphylococcus aureus to elastin. J Biol Chem 2004, 279:38433-38440.

42. Massey RC, Kantzanou MN, Fowler T, et al.: Fibronectinbinding protein A of Staphylococcus aureus has multiple, substituting, binding regions that mediate adherence to fibronectin and invasion of endothelial cells. Cell Microbiol 2001, 3:839-851.

43. Wann E, Gurusiddappa S, Höök M: The fibronectin-binding MSRAMM FnbpA of Staphylococcus aureus is a bifunctional protein that also binds to fibrinogen. $J$ Biol Chem 2000, 275:13863-13871.

44. Roche FM, Massey R, Peacock SJ, et al.: Characterization of novel LPXTG-containing proteins of Staphylococcus aureus identified from genome sequences. Microbiology 2003, 149(Pt 3):643-654.

45. Massey R, Dissanayeke S, Cameron B, et al.: Functional blocking of Staphylococcus aureus adhesins following growth in ex vivo media. Infect Immun 2002, 70:5339-5345.

46. Cheung $\mathrm{AL}$, Projan $\mathrm{SJ}$, Gresham $\mathrm{H}$ : The genomic aspect of virulence, sepsis, and resistance to killing mechanisms in Staphylococcus aureus. Curr Infect Dis Rep 2002, 4:400-410.

47. Cheung AL, Eberhardt KJ, Chung E, et al.: Diminished virulence of a sar-/agr- mutant of Staphylococcus aureus in the rabbit model of endocarditis. J Clin Invest 1994, 94:1815-1822.

48. Mayville P, Ji G, Beavis R, et al.: Structure-activity analysis of synthetic autoinducing thiolactone peptides from Staphylococcus aureus responsible for virulence. Proc Natl Acad Sci U S A 1999, 96:1218-1223. 
49. Entenza JM, Moreillon P, Senn MM, et al.: Role of sigmaB in the expression of Staphylococcus aureus cell wall adhesins ClfA and FnbA and contribution to infectivity in a rat model of experimental endocarditis. Infect Immun 2005, 73:990-998.
50. Bayer AS, Ramos MD, Menzies BE, et al.: Hyperproduction of alpha-toxin by Staphylococcus aureus results in paradoxically reduced virulence in experimental endocarditis: a host defense role for platelet microbicidal proteins. Infect Immun 1997, 65:4652-4660. 